\title{
Mechanism of lung injury caused by PM10 and ultrafine particles with special reference to COPD
}

\author{
W. MacNee, K. Donaldson
}

\begin{abstract}
Mechanism of lung injury caused by PM10 and ultrafine particles with special reference to COPD. W. MacNee, K. Donaldson. C) ERS Journals Ltd 2003.

ABSTRACT: Particulate air pollution (particles with a $50 \%$ cut-off aerodynamic diameter of $10 \mu \mathrm{m}$ (PM10)) has strong associations with the adverse health events detected by epidemiological studies in chronic obstructive pulmonary disease patients in diverse geographical locations. Transition metals may determine the toxic effects of PM10 through oxidative stress which may be injurious as shown by an increase in airspace epithelial permeability and may lead to inflammation through the activation of transcription factors for pro-inflammatory genes in both macrophages and epithelial cells. Recently, the present authors have shown that particulate air pollution may cause further molecular events that enhance transcription factor activation by causing acetylation of histones leading to unwinding of deoxyribonucleic acid (DNA) enhancing transcription factor DNA binding and increasing transcription for pro-inflammatory genes.

Further, ultrafine particles $(<\mathbf{1 0 0} \mathrm{nm}$ diameter), which have marked toxicity, may be responsible for some of the PM10 adverse effects. The current authors have demonstrated that ultrafine carbon black (ufCB) does not have its effects via transition metal-mediated mechanisms. However, ufCB and other ultrafines generate free radicals at their surface as measured by a number of chemical assays and are able to cause oxidative stress to cells and this is likely to be a factor in their ability to cause inflammation. Changes in calcium resulting from oxidative stress within cells may be an additional factor leading to transcription of pro-inflammatory genes.

Understanding the mechanisms of the harmful effects of particulate air pollution in chronic obstructive pulmonary disease may help in risk strategy for individuals who are susceptible to the effects of air pollution.

Eur Respir J 2003; 21: Suppl. 40, 47s-51s.
\end{abstract}

Edinburgh Lung and the Environment Group Initiative Colt Research Laboratories, Dept of Clinical and Radiological Sciences, University of Edinburgh, UK

Correspondence: W. MacNee

Edinburgh Lung and the Environment Group Initiative/Colt Research Laboratories Wilkie Building

Dept of Clinical and Radiological Sciences

University of Edinburgh

Medical School

Teviot Place

Edinburgh

UK

Fax: 441316511558

E-mail: w.macnee@ed.ac.uk

Keywords: Chronic obstructive pulmonary disease

elderly

fine particles

lung injury

ultrafine particles

Received and accepted: April 122002
The mammalian lung has evolved to clear particles provided that there is a low exposure to nontoxic particles, but some classes of particle cause problems for the normal deep lung clearance systems, leading to lung injury. These include the most well recognised pathogenic particles, such as asbestos and crystalline silica, whose mechanism of subversion of the normal phagocytic defences by damaging and activating lung cells are well documented. This paper deals with the mechanism of action of ultrafine particles in causing lung injury and avoiding normal clearance. Particles in environmental air are measured by the PM10 convention that measures the mass of airborne particles with 50\% efficiency for those with an aerodynamic diameter of $10 \mu \mathrm{m}$ in $\mu \mathrm{g} \cdot \mathrm{m}^{-3}$. Particles that are $<100 \mathrm{~nm}$ in diameter are commonly defined as ultrafine particles and are quantified within this convention [1]. Combustion-derived ultrafine particles, such as diesel soot, are the most numerous particles, by number, in urban PM10. Ultrafine particles are generated indoors by gas cooking and very high numbers can be produced. Diesel particles have an average singlet particle size of $20-40 \mathrm{~nm}$, and are present in aggregates (fig. 1) with associated sulphates, metals and hydrocarbons [3]. Singlet particles with aerodynamic diameters down to $10 \mathrm{~nm}$, deposit with high efficiency in the airways and centri-acinar regions of the lung [4].

\section{Particles and chronic obstructive pulmonary disease}

Amongst the elderly are susceptible individuals with chronic lung and cardiovascular disease, and so the elderly are a population that warrant studying. Aging is accompanied by changes in the functions of leukocytes and antioxidant defences and so the handling of particles by the lungs might change, including handling of ultrafines. Since the dominant hypothesis for the effects of ultrafines is focused on their ability to cause oxidative stress, then any lowering of the antioxidant defences in the elderly could predispose them to mount inappropriate and harmful inflammatory responses. Indoor air pollution may also be more important for the elderly since they spend more time indoors and so studies on
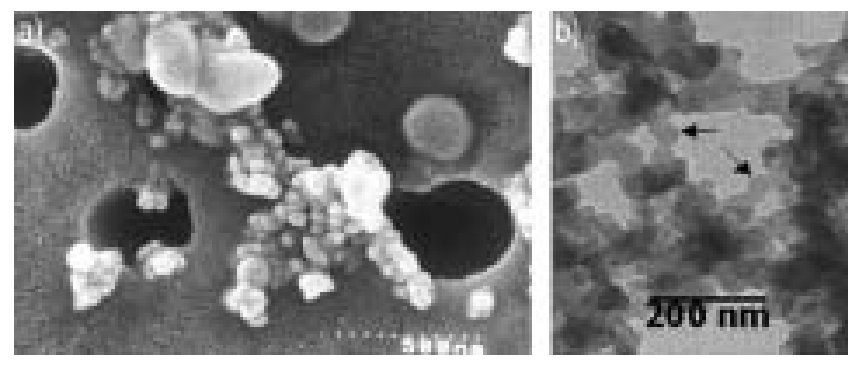

Fig. 1.-Aggregates of ultrafine particles in air from a) Birmingham (scanning electron microscope image, from the 3rd Quality of Urban Air Review Group (QUARG) report [2]) and b) Glasgow (Transmission electron microscope image courtesy of M. Wilson, School of Life Sciences, Napier University, Edinburgh, UK). In both case there are large aggregates composed of singlet ultrafine and larger particles. Scale bar is $500 \mathrm{~nm}$ in a) and $200 \mathrm{~nm}$ in b). 
indoor sources of ultrafines (such as gas cooker particles which preliminary experiments indicate may have the potential to cause inflammation) for their effects on the elderly should be considered, as an aid to risk management [5].

There is now considerable epidemiological evidence supporting the relationship between increased levels of particulate air pollution and increased morbidity and mortality from respiratory diseases [6]. There is a strong relationship between PM10 and exacerbations of chronic obstructive pulmonary disease (COPD) [7]. These associations are generally found at relatively low levels of ambient particulates, much lower than those found in occupational exposures or indeed in the high pollution episodes which occurred in the 1940s and 1950s. Exacerbations of COPD are among the commonest acute respiratory emergencies and result in considerable morbidity and mortality.

\section{Ultrafine particles}

Particles that are $<100 \mathrm{~nm}$ in diameter are commonly defined as ultrafine particles and these particles pose special problems for the lung. Singlet ultrafine particles are very small in comparison to the cellular structures and this may underlie the special toxicity of the ultrafines [1]. Although they readily form aggregates the fate of these aggregates in the lung is unknown and singlet particles may be released under the action of lung lining fluid. Ultrafine particles have a high surface area per unit mass compared to larger particles, even in aggregates, and the surface characteristics of particles are considered to be of central importance to their biological activity. Combustion-derived ultrafine particles, such as diesel soot, are the most numerous particles in urban PM10 (fig. 1).

Diesel particles are largely insoluble because of their particulate carbon core, with an average singlet particle size of 10-20 nm, and are present in aggregates with associated soluble sulphates, metals and hydrocarbons [3].

Singlet particles with aerodynamic diameters down to $10 \mathrm{~nm}$ deposit with high efficiency in the airways and centriacinar regions of the lung [1]. Aggregates will have a greater aerodynamic diameter than singlet particles and the aerodynamic characteristics of the aggregates will be dependent on the compactness of the aggregate. If the aggregate is more open with side chains etc. then it will have greater aerodynamic resistance, less likelihood of settling and hence a smaller aerodynamic diameter.

\section{Ultrafines in PM10}

Some components of PM10 are not very toxic, e.g. sulphates, nitrates, chlorides, ammonium and wind-blown crystal dust. Therefore toxicological attention has focused on the components of PM10 that are most likely to mediate adverse health effects and so the ultrafines have come to the fore in the so-called "ultrafine hypothesis" for the adverse effects of PM10 [8, 9]. However, transition metals and endotoxin are also potential mediators of the adverse health effects of PM10. Both PM10 and ultrafine carbon black (ufCB) cause more inflammation than the same instilled mass of fine carbon particles (control) (fig. 2) and ultrafine carbon causes more inflammation than PM10. The role that the ultrafine component of PM10 plays in the effects of PM10 on COPD patients is not known.

\section{Ability of ultrafine particles to injure the lungs}

In animal studies the acute inflammation caused by ultrafines is greater than the inflammation caused by fine particles at

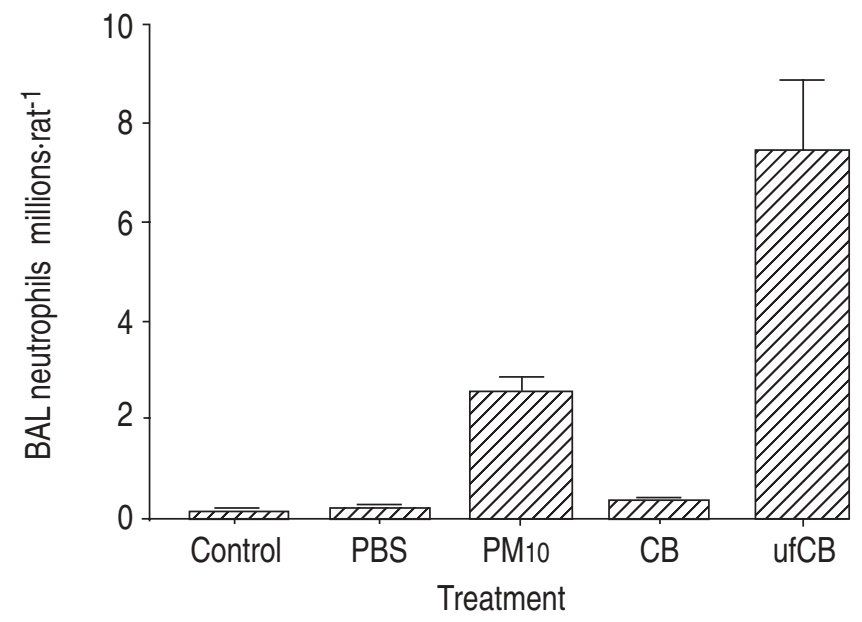

Fig. 2. - Inflammation caused by an equal instilled mass $(125 \mu \mathrm{g})$ of particles with a $50 \%$ cut-off aerodynamic diameter of $10 \mu \mathrm{m}$ (PM10), carbon black (CB) or ultrafine carbon black (ufCB). PBS: instilled with phosphate buffered saline; control: untreated; BAL: bronchoalveolar lavage.

equal mass [10]. This is directly related to the surface area lung burden of particulate as summarised in figure 3 where the inflammation caused by instillation of varying doses of fine and ultrafine particles composed of low toxicity materials is expressed as a function of the surface area dose instilled. As can be seen there is a direct relationship between the surface area of particles in the lung and the extent of the inflammatory response as measured by neutrophils in lavage.

Ultrafine particles also have important, growing industrial applications, for example ufCB, and so there is potential for occupational exposure. However, the extent of the risk to workers has not yet been quantified.

\section{Mechanism of the pro-inflammatory effects of ultrafine particles}

The mechanism of the inflammatory effects of ultrafine particles has been studied in a number of toxicological models.

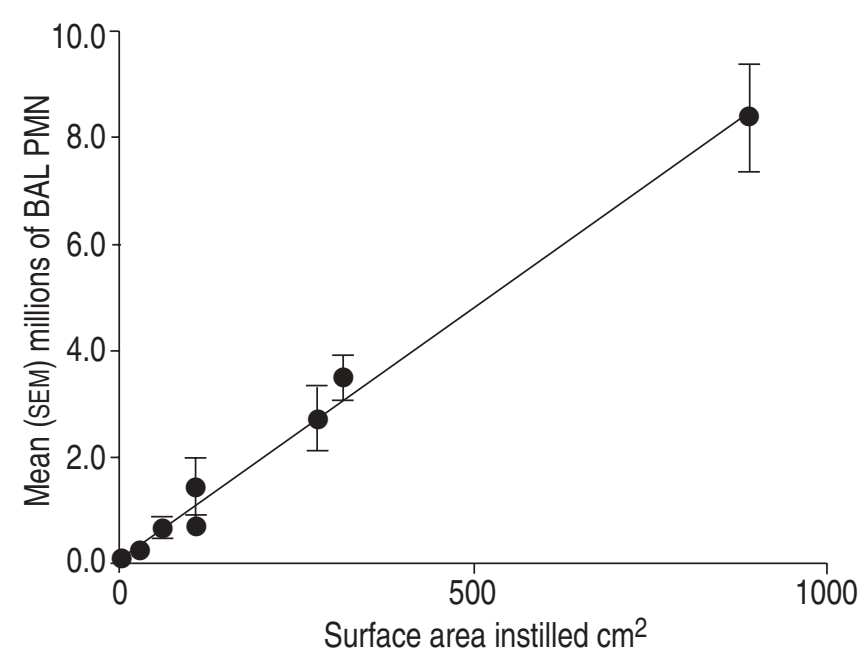

Fig. 3. - Inflammation at $24 \mathrm{~h}$ caused by instillation of various doses of fine and ultrafine titanium dioxide, carbon black and latex into the lungs of rats. Inflammation expressed as total polymorphonuclear neutrophil leukocyte (PMN) in lavage of three rats (mean \pm SEM) in relation to the total surface area of particles instilled. BAL: bronchoalveolar lavage. 


\section{Effects on phagocytosis}

Phagocytosis by alveolar macrophages is important in the clearance of particle from the lungs. In a study by the current authors [11] a macrophage cell line (J774.2) was exposed to ultrafine and fine samples of CB. The diameter of the singlet ultrafine was $\sim 20 \mathrm{~nm}$, although they were in aggregates. The diameter of the fine was $\sim 200 \mathrm{~nm}$, also in aggregates. The cells were then exposed to $2 \mu \mathrm{m}$ diameter indicator fluorescent latex beads and the uptake of these was used to judge phagocytic ability. UfCB had a more deleterious effect on phagocytosis of the latex indicator beads than CB. The effect was not apparently mediated by soluble mediators but by cellcell contact. Thus the adverse effects of ultrafine particles may be mediated in part by their ability to inhibit phagocytosis, allowing ultrafine particles and other particles that deposit along with them to persist unphagocytosed in the lung, stimulating epithelial cells and potentially gaining access to the interstitium.

\section{The role of oxidative stress}

The present authors have previously proposed a hypothesis for the mechanism of action of the adverse health effects of PM10 [10]. Central to this hypothesis is the generation of oxidative stress directly from the ultrafine and transition metal component of PM10 and indirectly from the recruitment to the airspaces and activation of blood leukocytes. Oxidative stress is considered to be a fundamental factor in the generation of inflammation through redox sensitive transcription factors, such as nuclear factor kappa B (NF-кB); the role of $\mathrm{NF}-\kappa \mathrm{B}$ and oxidative stress in inflammation in general, and in COPD in particular, is reviewed in reference [12].

The present authors have provided the following support for the role of oxidative stress in PM10-mediated inflammation. 1) Instillation of PM10 collected in central Edinburgh into the rat lung produces considerably more inflammation, as measured by bronchoalveolar lavage neutrophils, than an equal mass of fine ( $260 \mathrm{~nm}$ diameter) carbon particles, this is accompanied by a fall in bronchoalveolar lavage levels of reduced glutathione and an increase in oxidised glutathione [13]. 2) The influx of neutrophils following instillation of PM10 was significantly decreased by co-instillation with the thiol antioxidant Nacystelyn. 3) Direct generation of oxidants by PM10 has been shown in studies where free-radicals generated by PM10 can be quantified by their ability to cause injury to supercoiled plasmid deoxyribonucleic acid (DNA) [14]. 4) PM10 causes translocation of $N F-\kappa B$ from the cytoplasm to the nucleus in lung epithelial cells in vitro as demonstrated by a fluorescent antibody to the p50 component

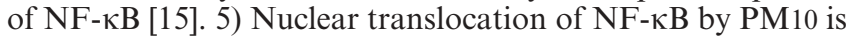
associated with activation of $\mathrm{NF}-\kappa \mathrm{B}$ as shown in studies of a stably transfected $\mathrm{NF}-\kappa \mathrm{B}$ luciferase reporter system in an alveolar epithelial cell line in vitro [15]; this activation of NF- $\kappa$ B by PM10 can be blocked by chelating transition metals. 6) Preliminary data from the current authors' laboratory has shown that PM10 can cause decreased gene expression for histone deacetylase 2 in alveolar epithelial cells, which can be shown to cause increased acetylation of histone residues allowing increased transcription factor binding and enhanced transcription for pro-inflammatory cytokines such as interleukin (IL)-8. This effect of PM10 in enhancing histone acetylation can be prevented by pre-treatment of cells with antioxidants.

Taken together these data suggest that the generation of oxidative stress in the airspaces on exposure to PM10 could enhance the already increased oxidant burden which occurs in the lungs of patients with COPD [16]. This oxidative stress can cause transcription of pro-inflammatory genes via $\mathrm{NF}-\kappa \mathrm{B}$ activation and histone acetylation (fig. 4). During exacerbations of the disease there is evidence of systemic oxidative stress, as measured by enhanced release of reactive oxygen species from peripheral blood leukocytes and a decrease in plasma antioxidant capacity [17].

\section{Potential role of ultrafine particles in PM10}

PETERs et al. [18] reported that decrements in evening peak flow in a group of asthmatics during a severe air pollution episode, were best associated with the ultrafine component of the airborne particles.

Ultrafine $\mathrm{CB}$ has been reported to rapidly stimulate the opening of membrane calcium channels in macrophages leading to a substantial increase in the intracellular calcium levels [19-21]. The role that these calcium fluxes play in the inhibition of phagocytosis remain to be investigated but increased calcium stimulates transcription of pro-inflammatory genes in macrophages and in epithelial cells if the same effects are seen [22]. In subsequent experiments, blocking the membrane calcium $(\mathrm{Ca})^{2+}$ channels with verapamil has blocked the ability of ufCB to stimulate the release of tumour necrosis factor $\alpha$ (unpublished data).

\section{Infection and susceptibility to PM10 in COPD patients}

RETAMALES et al. [23] have described greater expression of adenoviral early region $1 \mathrm{~A}$ (E1A) genes in the lungs of smokers who have developed COPD, than in smokers with the same smoking history who have not developed the disease. Thus a hypothesis has been advanced that persistent viral infection through this mechanism could be a susceptibility factor for the development of COPD.

The E1A protein assists viral replication by interacting with host cell DNA binding proteins such as NF- $\kappa \mathrm{B}$ and activator protein 1. E1A DNA can persist following acute viral

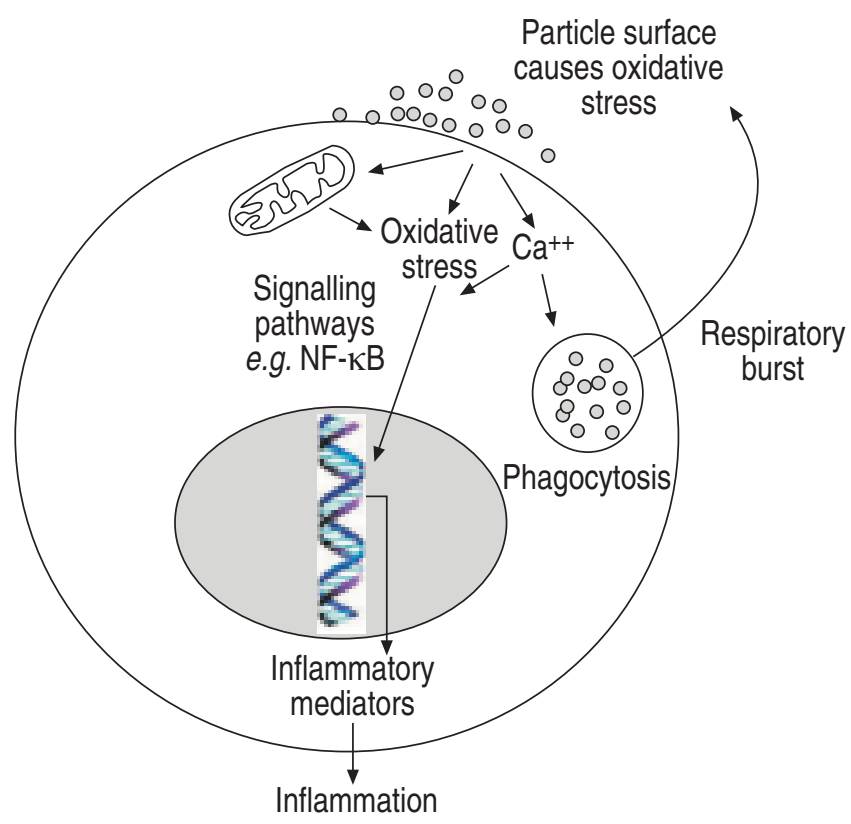

Fig. 4. - Hypothetical sequence of events leading from the contact of particles with the cells (composite macrophage/epithelial cell) surface to inflammation. NF-кB: nuclear factor $\kappa \mathrm{B}$; Ca: calcium. 
Airways in stable asthma and COPD

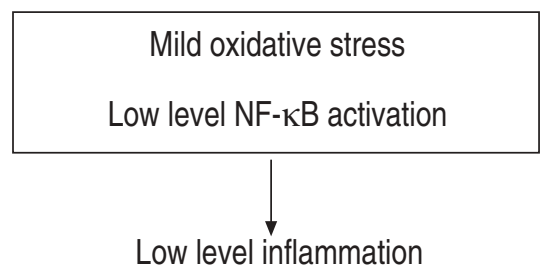

\section{Asthma and COPD airways \\ following exposure to increased PM10}

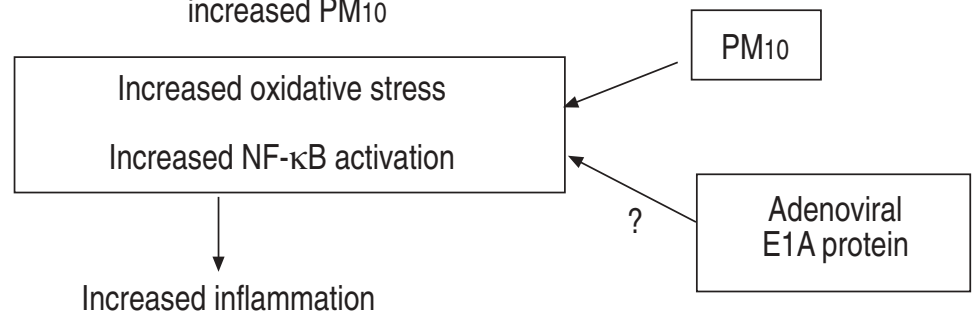

Exacerbation

Fig. 5. - Hypothetical interaction between particles with a 50\% cut-off aerodynamic diameter of $10 \mu \mathrm{m}$ (PM10) and inflamed airways leading to

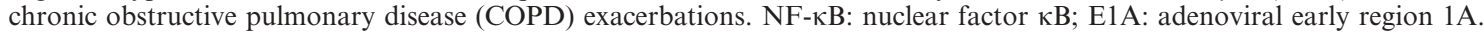

infection in many cells in the respiratory tract including epithelial cells. During latent expression of the E1A gene there is therefore the potential for disregulation of genes that rely on NF- $\kappa \mathrm{B}$ and other transcription factors for pro-inflammatory mediators. In response to PM10, alveolar epithelial cells upregulate IL-8 messenger ribonucleic acid (mRNA) and release increased IL-8 protein. In an in vitro model of latent adenoviral infection, in which the E1A gene is transfected into alveolar epithelial cells, there is far greater IL-8 mRNA expression in response to PM10 [24]. Thus both acute viral infection, interacting with PM10 or the presence of latent adenoviral infection, could enhance the pro-inflammatory effects of PM10 inhalation (fig. 5).

\section{Future research}

The in vivo and in vitro animal experiments described here suggest that the mechanisms of the pro-inflammatory effects of PM10 involve oxidative stress, upregulation of transcription factors and pro-inflammatory gene expression, but need to be tested in human exposures. Newer techniques are now available to allow these mechanisms to be tested. The presence of oxidative stress following exposure to pollutants can be assessed systemically in peripheral blood or in breath condensate, such as by measuring 8-isoprostane a lipid peroxidation product of arachidonic acid [25]. Inflammatory responses in the airways such as neutrophil influx, cytokine (IL-8, TNF) levels can be measured non-invasively in induced sputum [24]. Cells from induced sputum can be assessed for transcription factor activation, gene expression and even histone acetylation/deacetylation [27, 28]. The mechanisms described in this paper can now be tested in normal subjects and susceptible individuals such as patients with COPD exposed to particulates for example from diesel exhaust or indoor air pollutants such as particulates from gas cooking. The study of these mechanisms, in human exposures should help in risk management in susceptible individuals and also may lead to interventions, which may prevent exacerbations of COPD produced by air pollution.

\section{Conclusions}

This short review summarises the role of increases in particles with a $50 \%$ cut-off aerodynamic diameter of $10 \mu \mathrm{m}$ and especially the ultrafine particle component, in exacerbations of chronic obstructive pulmonary disease. Ultrafine particles and transition metals are common components of particles with a $50 \%$ cut-off aerodynamic diameter of
$10 \mu \mathrm{m}$ that cause oxidative stress and this may enhance pro-inflammatory effects in airways of chronic obstructive pulmonary disease patients that are already inflamed by their disease. Infection with adenovirus and other pathogens may also interact with oxidative stress and particles to promote exacerbations.

\section{References}

1. Donaldson K, Stone V, Clouter A, Renwick L, MacNee W. Ultrafine particles. Occup Environ Med 2001; 58: 211-216.

2. Quality of Urban Air Review Group. Airborne particulate matter in the United Kingdom: third report of the Quality of Urban Air Review Group. Quality of Urban Air Group, 1996.

3. Kittelson DB. Engines and nanoparticles: a review. J Aerosol Sc 1998; 29: 575-588.

4. Kim CS, Jaques P. Respiratory dose of inhaled ultrafine particles in healthy adults. Phil Trans $R$ Soc Lond A 2000; 358: 2693-2705.

5. Dick CA, Dennekamp M, Howarth S, et al. Stimulation of IL-8 release from epithelial cells by gas cooker PM(10): a pilot study. Occup Environ Med 2001; 58: 208-210.

6. Pope CA, Dockery DW. Epidemiology of particle effects. In: Holgate ST, Samet JM, Koren HS, Maynard RL, eds Air Pollution and Health. San Diego, Academic Press, 1999; 673-705.

7. Pope CA. Epidemiology of fine particulate air pollution and human health: biologic mechanisms and who's at risk? Environ Health Perspect 2000; 108: 713-723.

8. MacNee W, Donaldson K. Particulate air pollution: injurious and protective mechanisms in the lungs. In: Holgate ST, Samet JM, Koren HS, Maynard RL, eds. Air Pollution and Health. San Diego, Academic Press, 1999; 653-672.

9. Utell MJ, Frampton MW. Acute health effects of ambient air pollution: the ultrafine particle hypothesis. J Aerosol Med 2000; 13: 355-359.

10. Donaldson K, Stone V, MacNee W. The toxicology of ultrafine particles. In: Maynard RL, Howards CV, eds. Particulate matter: properties and effects upon health. Oxford, Bios, 1999; 115-127.

11. Renwick LC, Donaldson K, Clouter A. Impairment of alveolar macrophage phagocytosis by ultrafine particles. Toxicol Appl Pharmacol 2001; 172: 119-127.

12. MacNee W, Rahman I. Is oxidative stress central to the pathogenesis of chronic obstructive pulmonary disease? Trends Mol Med 2001; 7: 55-62.

13. Li XY, Gilmour PS, Donaldson K, MacNee W. Free radical activity and pro-inflammatory effects of particulate air pollution (PM10) in vivo and in vitro. Thorax 1996; 51: 1216-1222. 
14. Gilmour PS, Brown DM, Lindsay TG, Beswick PH, MacNee W, Donaldson K. Adverse health effects of PM10 particles: involvement of iron in generation of hydroxyl radical. Occup Environ Med 1996; 53: 817-822.

15. Jimenez LA, Thompson J, Brown DA, et al. Activation of NF-kappa B by PM(10) occurs via an iron-mediated mechanism in the absence of IkappaB degradation. Toxicol Appl Pharmacol 2000; 166: 101-110.

16. Rahman I, MacNee W. Oxidant antioxidant imbalance in smokers and chronic obstructive pulmonary-disease. Thorax 1996; 51: 348-350.

17. Rahman I, MacNee W. Role of oxidants/antioxidants in smoking-induced lung-diseases. Free Rad Biol Med 1996; 21: 669-681.

18. Peters A, Wichmann HE, Tuch T, Heinrich J, Heyder J. Respiratory effects are associated with the number of ultrafine particles. Am J Respir Crit Care Med 1997; 155: 1376-1383.

19. Stone V, Shaw J, Brown DM, MacNee W, Faux SP, Donaldson $\mathrm{K}$. The role of oxidative stress in the prolonged inhibitory effect of ultrafine carbon black on epithelial cell function. Toxicol in Vitro 1998; 12: 649-659.

20. Stone V, Tuinman M, Vamvakopoulos JE, et al. Increased calcium influx in a monocytic cell line on exposure to ultrafine carbon black. Eur Respir J 2000; 15: 297-303.

21. Dolmetsch RE, Lewis RS, Goodnow CC, Healy JI. Differential activation of transcription factors induced by $\mathrm{Ca} 2+$ response amplitude and duration. Nature 1997; 386: 855-858.
22. Donaldson K, Stone V, Gilmour PS, Brown DM, MacNee W. Ultrafine particles: mechanisms of lung injury. Phil Trans $R$ Soc Lond A 2000; 358: 2741-2749.

23. Retamales I, Elliott WM, Meshi B, et al. Amplification of inflammation in emphysema and its association with latent adenoviral infection. Am J Respir Crit Care Med 2001; 164 : 469-473.

24. Gilmour PS, Rahman I, Hayashi S, Hogg JC, Donaldson K, MacNee W. Adenoviral E1A primes alveolar epithelial cells to PM(10)-induced transcription of interleukin-8. Am J Physiol Lung Cell Mol Physiol 2001; 281: L598-L606.

25. Montuschi P, Collins JV, Ciabattoni G, et al. Exhaled 8-isoprostane as an in vivo biomarker of lung oxidative stress in patients with COPD and healthy smokers. Am J Respir Crit Care Med 2000; 162: 1175-1177.

26. Keatings VM, Collins PD, Scott DM, Barnes PJ. Differences in interleukin-8 and tumor-necrosis-factor-alpha in induced sputum from patients with chronic obstructive pulmonary disease or asthma. Am J Respir Crit Care Med 1996; 153: 530-534.

27. Rahman I, MacNee W. Histone deacetylase expression in sputum leucocytes may be associated with cytokine generation in COPD. Am J Respir Crit Care Med 2001; 163: A908.

28. Drost EM, Gilmour PS, Ritchie H, et al. Histone deacetylase expression in sputum leucocytes may be associated with cytokine generation in COPD. Am J Respir Crit Care Med 2001; 163: A908. 